\title{
Multimodal breast cancer imaging using coregistered dynamic diffuse optical tomography and digital breast tomosynthesis
}

Bernhard B. Zimmermann

Bin Deng

Bhawana Singh

Mark Martino

Juliette Selb

Qianqian Fang

Amir Y. Sajjadi

Jayne Cormier

Richard H. Moore

Daniel B. Kopans

David A. Boas

Mansi A. Saksena

Stefan A. Carp 


\title{
Multimodal breast cancer imaging using coregistered dynamic diffuse optical tomography and digital breast tomosynthesis
}

\author{
Bernhard B. Zimmermann, ${ }^{\mathrm{a}, \mathrm{b}}$ Bin Deng, ${ }^{\mathrm{a}, \mathrm{c}}$ Bhawana Singh, ${ }^{\mathrm{a}, \mathrm{c}}$ Mark Martino, ${ }^{\mathrm{a}}$ Juliette Selb, ${ }^{\mathrm{a}, \mathrm{c}}$ Qianqian Fang, ${ }^{\mathrm{d}}$ \\ Amir Y. Sajjadi, ,a, Jayne Cormier, ${ }^{e}$ Richard H. Moore, ${ }^{e}$ Daniel B. Kopans, ,,e David A. Boas, ${ }^{\text {a,c }}$ \\ Mansi A. Saksena, ${ }^{\mathrm{c}, \mathrm{e}}$ and Stefan A. Carp ${ }^{\mathrm{a}, \mathrm{c}, *}$ \\ aMassachusetts General Hospital, Athinoula A. Martinos Center for Biomedical Imaging, Department of Radiology, Charlestown, \\ Massachusetts, United States \\ ${ }^{b}$ Massachusetts Institute of Technology, Department of Electrical Engineering and Computer Science, Cambridge, Massachusetts, United States \\ 'Harvard Medical School, Department of Radiology, Boston, Massachusetts, United States \\ ${ }^{d}$ Northeastern University, Department of Bioengineering, Boston, Massachusetts, United States \\ 'Massachusetts General Hospital, Breast Imaging Division, Department of Radiology, Boston, Massachusetts, United States
}

\begin{abstract}
Diffuse optical tomography (DOT) is emerging as a noninvasive functional imaging method for breast cancer diagnosis and neoadjuvant chemotherapy monitoring. In particular, the multimodal approach of combining DOT with x-ray digital breast tomosynthesis (DBT) is especially synergistic as DBT prior information can be used to enhance the DOT reconstruction. DOT, in turn, provides a functional information overlay onto the mammographic images, increasing sensitivity and specificity to cancer pathology. We describe a dynamic DOT apparatus designed for tight integration with commercial DBT scanners and providing a fast (up to $1 \mathrm{~Hz}$ ) image acquisition rate to enable tracking hemodynamic changes induced by the mammographic breast compression. The system integrates 96 continuous-wave and 24 frequency-domain source locations as well as 32 continuous wave and 20 frequency-domain detection locations into low-profile plastic plates that can easily mate to the DBT compression paddle and $\mathrm{x}$-ray detector cover, respectively. We demonstrate system performance using static and dynamic tissue-like phantoms as well as in vivo images acquired from the pool of patients recalled for breast biopsies at the Massachusetts General Hospital Breast Imaging Division. ๑ 2017 Society of Photo-Optical Instrumentation Engineers (SPIE) [DOI: 10.1117/1.JBO.22.4.046008]
\end{abstract}

Keywords: diffuse optical tomography; dynamic imaging; breast cancer; digital breast tomosynthesis; multimodal; optical mammography.

Paper 160848R received Dec. 15, 2016; accepted for publication Apr. 7, 2017; published online Apr. 27, 2017.

\section{Introduction}

The American Cancer Society estimates that in 2016 246,660 women will be diagnosed with invasive breast cancer in the USA alone. ${ }^{1}$ When excluding nonmelanoma skin cancer, the breast is the most common cancer site in females with a share of $29 \%$ of new cases, and the overall lifetime probability of developing breast cancer is estimated at $12.3 \% .^{1}$ Even though the survival rate has significantly increased in the last 40 years, earlier detection and improved treatment remain a priority.

$\mathrm{X}$-ray mammography and its extension to three-dimensional (3-D) imaging, digital breast tomosynthesis (DBT), are the most widely used screening modalities and have been attributed to a $14 \%$ to $32 \%$ relative reduction in breast cancer mortality for women aged 39 to 69 years. $^{2}$ Both of these techniques can offer greater than $80 \%$ sensitivity, ${ }^{3-5}$ but significant difficulties in women with dense breasts ${ }^{6}$ and poor specificity in clinical use, ${ }^{7-9}$ resulting in benign biopsies over $70 \%$ of the time, ${ }^{10}$ remain a challenge. These biopsies that ultimately show no evidence of cancer can cause significant stress to the patients and their families.

Efforts to overcome these shortcomings are being made in multiple areas. DBT, due to its 3-D imaging of the breast, is

*Address all correspondence to: Stefan A. Carp, E-mail: carp@nmr.mgh .harvard.edu able to offer moderately increased sensitivity and specificity by reducing the confusing superposition of layers of breast tissue present in traditional digital mammography. ${ }^{11-15}$ Furthermore, functional methods, such as contrast-enhanced magnetic resonance imaging (MRI) ${ }^{16-20}$ and positron emission tomography $(\mathrm{PET}),{ }^{21-23}$ are gaining ground with respect to specificity. ${ }^{24-28}$ Unfortunately, the cost and availability of these technologies and the injection of tracers ${ }^{29}$ prevent them from wide adoption for screening exams.

Near-infrared (NIR) diffuse optical tomography (DOT), an emerging technology for tissue functional imaging, can offer a relatively inexpensive, noninvasive, and nonionizing alternative to the high-cost methods mentioned above. DOT works by sending NIR light along multiple paths through tissue, and in the simplest case measuring the attenuation of a continuous-wave (CW) light source, or additionally the phase shift of a radiofrequency (RF) power-modulated light source. Alternatively, the attenuation and broadening in time domain (TD) of a pulsed light source can be measured. ${ }^{30} \mathrm{RF}$ and TD measurements allow the disentanglement of absorptive and scattering effects. By fitting a light diffusion model to measurements acquired from source and detector arrays that at least partially encompass the tissue, localized absorption and scattering coefficients can be reconstructed. Finally, by combining results from two or

1083-3668/2017/\$25.00 @ 2017 SPIE 
more wavelengths, 3-D chromophore concentration maps can be obtained.

Innovative DOT-based optical breast imaging systems having circular, ${ }^{31,32}$ pentagonal, ${ }^{33}$ cup shaped, ${ }^{34}$ as well as parallel plate ${ }^{35-37}$ optode geometries have been reported. Inspired by the success of other multimodal imaging technologies, especially PET combined with computed tomography, researchers began to explore the possibility of combining DOT with structural imaging modalities for imaging breast cancer. Combining lowresolution functional imaging with high-resolution structural imaging in a spatially/temporally coregistered manner creates a win-win strategy: on the one hand, utilizing the high-resolution structure images as a prior, the functional imaging modality can yield improved image quality and reduced artifacts ${ }^{38,39}$ to deliver more accurate representation of the functional status of tissue; on the other hand, specificity of the structural imaging modalities can be improved by adding complementary physiological information from the functional imaging modality. Moreover, coregistration of two modalities can facilitate the extrapolation of image findings interpreted from one modality to the other, and ease in the acceptance of new technologies by the radiology community. Finally, functional imaging methods are especially suited for monitoring neoadjuvant chemotherapy, as changes in underlying tumor physiology are known to manifest earlier than the actual tumor shrinkage, ${ }^{40,41}$ which is the predominant metric used to assess tumor response in current clinical practice.

Coregistration of DOT with ultrasound ${ }^{42}$ and $\mathrm{MRI}^{43}$ has been investigated by various groups. However, combining DOT with DBT or $\mathrm{x}$-ray mammography is more promising in terms of clinical translation due to the high-prevalence, fairly low-cost, and high-resolution nature of these $\mathrm{x}$-ray technologies. Several studies have been published, ${ }^{44-46}$ including the results from our own first generation tomographic optical breast imager. ${ }^{47-49}$ In recent years, it has been discovered that monitoring dynamic changes in the breast due to breath maneuvers, ${ }^{50,51}$ gas inhalation, ${ }^{52}$ or mechanical stimulation ${ }^{53-57}$ can yield valuable additional information. Evaluating dynamic contrast due to mechanical stimulation in combined DBT and DOT systems is particularly efficient, because the breast will be readily under compression during mammography in any case.

Our group has already investigated hemodynamic responses of breasts under partial or full mammographic compression using a stand-alone DOT system, finding statistically different pressure responses of tumor tissue compared to healthy tissue. ${ }^{58-60}$ To take full advantage of this promising contrast mechanism, we built a second generation optical-DBT system (TOBI2) with dynamic imaging in mind. The main benefit of TOBI2 over the previous system ${ }^{48,49}$ is a much higher acquisition speed achieved by using a combination of frequency encoding, fast source switching, and parallel detection. Overall, up to 34 lasers can be powered at the same time, and all 52 detectors can be acquired simultaneously. Additional advantages of the new system are an increased optode density, which results in higher image resolution, as well as x-ray translucent source and detector plates. Employing x-ray translucent optical probes eliminates the necessity to remove them before $\mathrm{x}$-ray imaging, and thus enables true simultaneous dual-modal imaging and reduces the time spent in compression compared to sequential optical and x-ray imaging, thereby increasing patient comfort.

In this paper, we first describe the technical and instrumentation aspects of our TOBI2 system in detail. Then we demonstrate the performance of TOBI 2 in recovering optical contrasts, especially in dynamic imaging, with phantom measurements as well as initial patient images obtained with the system.

\section{Methods}

\subsection{System Overview}

The complete TOBI 2 system, together with its schematic drawing, is shown in Figs. 1 and 2, respectively. The optical probes are directly attached to the Hologic Selenia dimensions DBT machine and connected via glass fibers to the instrument tower. The tower houses both the RF and CW subsystems. Due to the necessity to locate TOBI 2 in an active clinical space, the tower is fully enclosed. On the outside of the tower, there is a shelf with the laptop controlling the optical system, as well as holders for
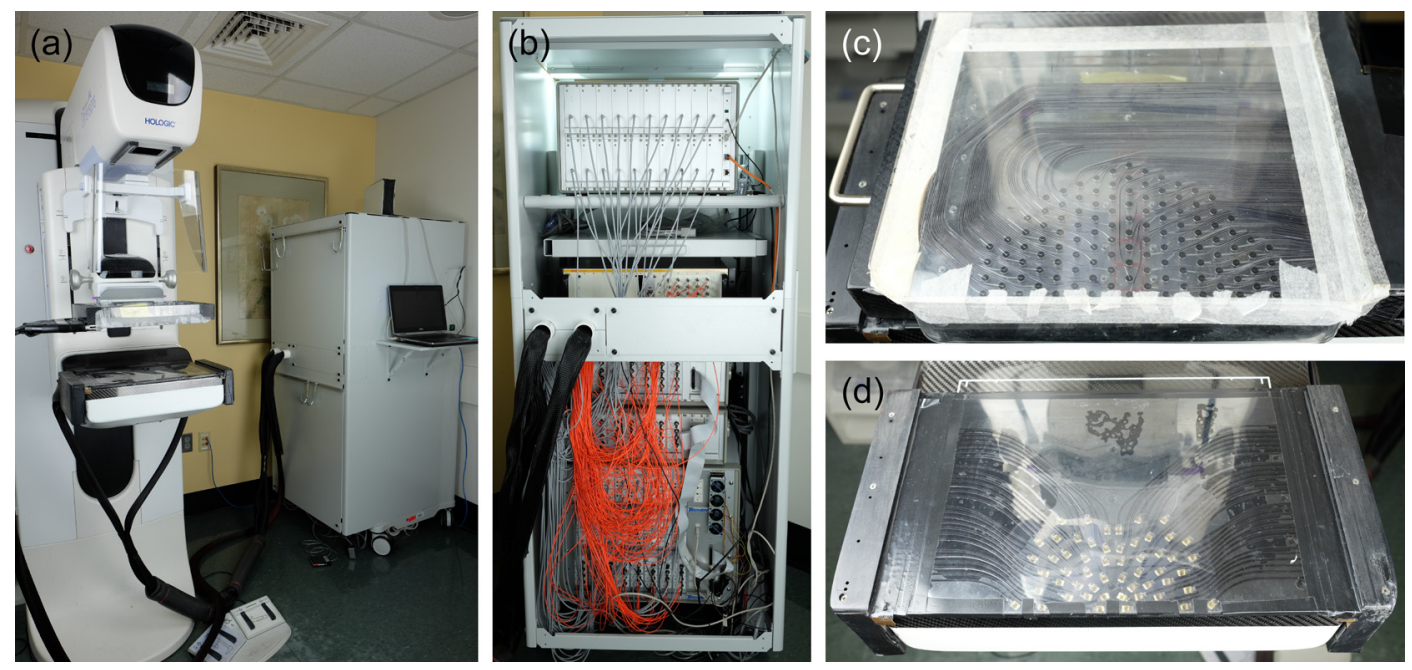

Fig. 1 (a) Complete TOBI2 system. Optical probe is attached to the DBT machine. Optical fiber bundles connect the optodes in the probe to the instruments inside the enclosed tower. (b) Inside view of the instrument tower. From top to bottom: the RF detectors, RF sources, $(2 \times)$ CW source expansion boxes, and the CW6 main instrument. (c) Close-up view of the source plate. (d) Close-up view of the detector plate. 


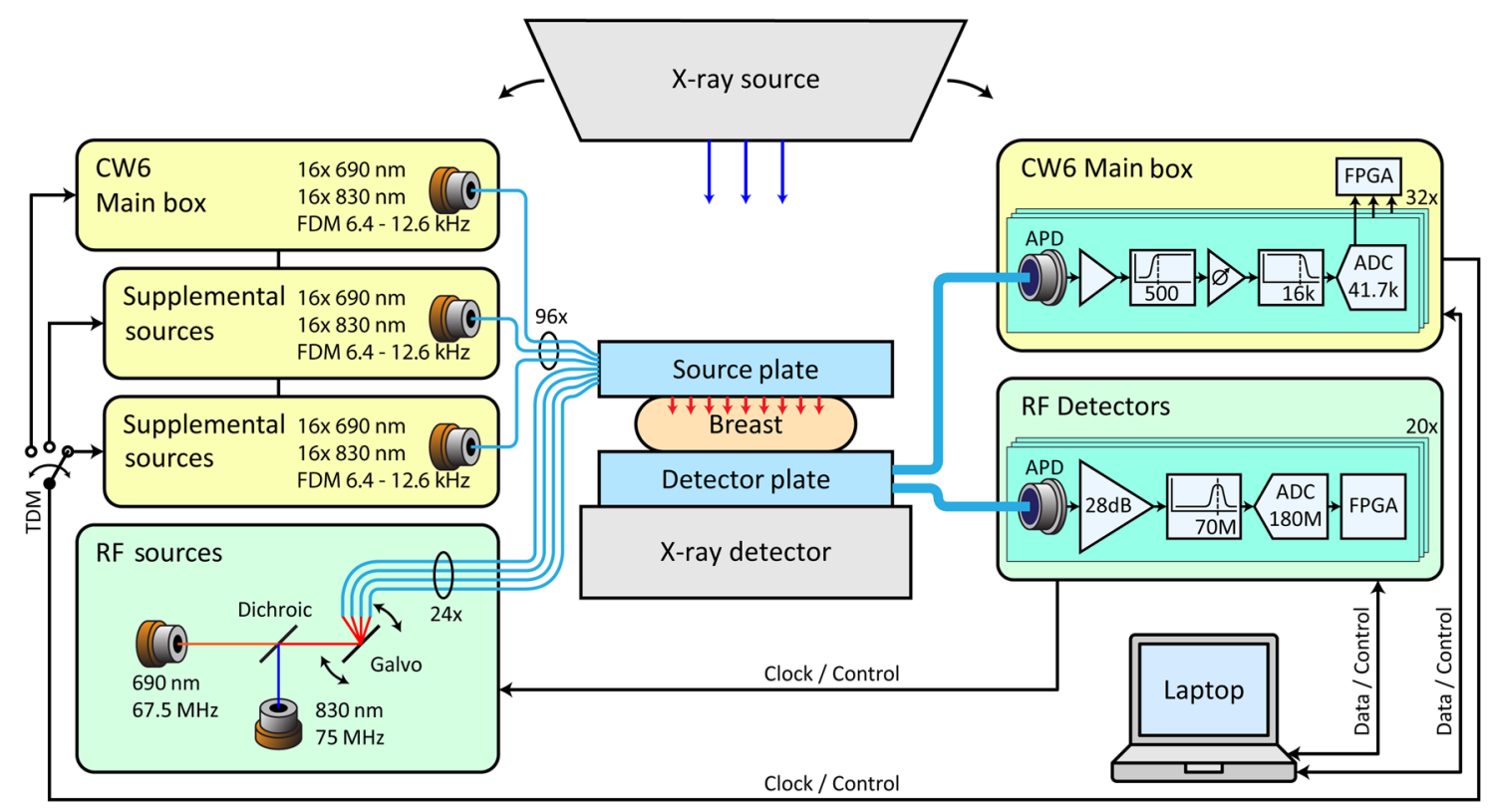

Fig. 2 Schematic overview of the TOBI2 system. CW components are shaded in yellow, RF components are shaded in light green, DBT system components are shaded in gray, and fiber optics are represented in blue.

the optical probe allowing storage when the DBT machine is used alone. In the following paragraphs, we describe each component in more detail.

\subsection{CW Component}

The CW imaging unit, shown in Fig. 1(b), was manufactured by TechEn Inc. (Milford, Massachusetts). It consists of the main CW6 system, containing 32 detectors and 32 lasers diodes split evenly between the wavelengths of 690 and $830 \mathrm{~nm}$. The unit also contains two supplemental boxes, each containing 32 lasers split again evenly between 690 and $830 \mathrm{~nm}$, for a total of $96 \mathrm{CW}$ lasers. Each laser is modulated with a square wave at one of 32 discrete frequencies between 6.4 and $12.6 \mathrm{kHz}$. The frequencies are chosen to span less than one octave, so that harmonics do not fall on other modulation frequencies. The system powers the lasers in the main CW6 unit and the supplemental boxes in a sequential, electronically switched order, so that only 32 lasers are on simultaneously. The dwell time in each state is specified in multiples of $40 \mathrm{~ms}$, and for the data shown in this paper, we used a dwell time of $1 \mathrm{~s}$, thus resulting in a frame rate of $1 / 3 \mathrm{~Hz}$.

On the detection side, each channel consists of a Hamamatsu avalanche photo diode (APD) module (C5460-01) followed by programmable signal amplification, conditioning, and digitization. On board field programmable gate arrays (FPGAs) and digital signal processors demodulate the signals by calculating the fast Fourier transform (FFT) of each detection channel and sending the raw intensity data of the appropriate FFT bin to the controlling computer via a universal serial bus (USB) connection at a rate of 25 samples/s.

\subsection{Radio Frequency Component}

The RF imaging unit, shown in Fig. 1(b), was built at the Martinos Center in collaboration with TechEn Inc. and was described before in Ref. 61. It contains one 685-nm laser diode modulated at 67.5 MHz, and one 830-nm laser diode modulated at $75 \mathrm{MHz}$. Both diodes are set to an average power of $\sim 25 \mathrm{~mW}$ and a modulation depth of $90 \%$. The light of these lasers is collimated and then combined with a dichroic mirror. The resulting dual wavelength beam is launched via a twodimensional galvo into one of 24 fibers. In the data shown here, the dwell time at each location is set to $0.13 \mathrm{~s}$ for the phantom measurements and $0.4 \mathrm{~s}$ for the patient measurement, resulting in cycle times over all 24 locations of 3.3 and $10 \mathrm{~s}$, respectively.

On the detector side, each of the 20 channels consists of a Hamamatsu APD module (C5331-04) followed by amplification and filtering. The signal of each channel is directly digitized at 180 million samples/s without down conversion by a 16-bit analog to digital converter (ADC). Each ADC has an FPGA attached that computes overlapping 4 million point discrete Fourier transforms to demodulate the signals, resulting in a final data rate of $90 \mathrm{~Hz}$ per wavelength. The data from all detector channels are collected and sent to a computer via USB by a control card.

\subsection{Optical Probe}

The optical probe consists of a source plate, shown in Fig. 1(c), which is permanently attached to the compression paddle of the DBT machine, and a detector plate, shown in Fig. 1(d), which fits on the X-ray detector cover of the DBT machine. The source plate contains $120500-\mu$ m poly (methyl methacrylate) (PMMA) fibers, placed individually in milled channels in a $1 / 8$-in. thick clear polycarbonate plate. At the edge of the source plate, the PMMA fibers are coupled into 500- $\mu \mathrm{m}$ glass fibers, which transport the source light from the $\mathrm{CW}$ and RF imaging units. At the light emitting ends, the PMMA fibers are polished at 45-deg angles to send light into the breast tissue. The fiber channels end within 5-mm black plastic disks to prevent light leakage.

The detector plate consists of a quarter inch thick black acrylonitrile butadiene styrene (ABS) plate, into which 54 
channels containing 2-mm PMMA fibers are milled. On the light collection side, the fibers are terminated with plastic prisms, and at the edge of the detector plate they are coupled into $2.5-\mathrm{mm}$ diameter glass fiber bundles that route the light back into the instrument tower and to the detectors.

The optode locations on the source and detector plates were chosen to give full breast coverage in $80 \%$ of patients, as determined by breast outlines obtained from DBT images from our previous study. ${ }^{49}$ Assignment of the source and detector locations to the CW and RF subsystems as well as to a specific wavelength has then been optimized using a genetic algorithm. Both the source and detector plates do not contain any glass or metal parts within the field of view of the DBT system to achieve x-ray translucency and minimize x-ray contrast.

\subsection{Phantom}

To measure the temporal and spatial resolution of TOBI2, we created two phantoms. The first phantom features one centrally located spherical cavity with a diameter of $19 \mathrm{~mm}$. The second phantom features three spherical cavities of 13-, 19-, and 25-mm diameter separated by $45 \mathrm{~mm}$. The cavities can be filled with liquids of varying optical properties through the channels embedded in the phantom to either match with or to create contrast to background optical properties for imaging.

Each phantom was constructed from $2.8 \mathrm{~L}$ of silicone (Smooth-On Ecoflex 00-50) mixed with $2660 \mathrm{mg}$ of white pigment and $78 \mathrm{mg}$ of black pigment (Smooth-On Silc Pig White \& Black). To avoid light piping, the inclusions were made without glass or any other material that could distort our results. Specifically, as seen in Fig. 3(a), the inclusions were made of water-soluble wax spheres (Freeman Sol-U-Carv) and were mounted in the mold with 2.4-mm diameter steel tubes. After pouring and curing of the degassed silicone, the tubes were removed, and the wax spheres were dissolved by flowing warm water through the channels. The finished phantoms, as shown in Fig. 3(b), have a thickness of $52 \mathrm{~mm}$. The size and separation of inclusions were further confirmed by DBT x-ray control images, shown in Fig. 3(c) for the triple-inclusion phantom.

\subsection{Patient Imaging}

A 47-year-old non-Hispanic white female with a breast cancer diagnosis was imaged on our system. An ultrasound guided left breast core biopsy indicated the presence of grade 3 invasive ductal carcinoma at the 5 o'clock position, located $3 \mathrm{~cm}$ from the nipple, measuring $1.8 \times 1.2 \times 1.1 \mathrm{~cm}^{3}$. An axillary lymph node core biopsy found lymph nodes with metastatic ductal carcinoma. Patient consent was obtained in accordance with the policies and guidelines of the Massachusetts General Hospital/Partners Healthcare Institutional Review Board. The subject's breast was imaged under partial compression (half mammographic force, $21.8 \mathrm{~N}$ for this patient) first, followed by imaging under full mammographic compression $(44.5 \mathrm{~N}$ for this patient). The imaging session lasted $\sim 3 \mathrm{~min}$.

\subsection{Image Reconstruction Methods}

Optical image reconstructions are performed on a pair of tetrahedral meshes, a finer one for solving the optical forward problem and a coarser one for solving the inversion, generated using the MATLAB-based meshing toolbox "iso2mesh." 62 A slab geometry is used to generate phantom meshes, whereas 3-D DBT images are used to extract the breast shape for patient scans. Raw optical measurements are first calibrated against a homogenous phantom with known optical properties and then fitted for bulk properties. Nonlinear, spectrally constrained inversion of the finite-element representation of the diffusion approximation using the Tikhonov-regulated Gauss-Newton approach is performed for nine iterations using our in-house software, i.e., Redbird, ${ }^{48}$ to reconstruct optical images shown in this paper. When solving the inverse problem, compositional structural priors are used as soft constraints in our structuralprior guided reconstruction algorithm described previously. ${ }^{63}$ In phantoms, a sphere located at the inclusion center, and with its diameter matched, is used to derive the structural prior for each phantom inclusion, and the prior for background is set to enforce unity of both tissue compositions on each mesh node. For patients, a dual-Gaussian segmentation algorithm ${ }^{63}$ is used to automatically derive adipose and fibroglandular compositional priors from DBT images. Similar to the phantom inclusion prior, a Gaussian sphere profile is used to generate an additional lesion prior with known centroid and size information provided by an experienced radiologist (Saksena).

\section{Results}

\subsection{System Characterization}

The RF component has already been characterized in Ref. 61. Here we will briefly summarize these findings. We measured a noise equivalent power of less than $1.4 \mathrm{pW} / \sqrt{ } \mathrm{Hz}$, which approaches the manufacturer specified noise floor of the APD module of $0.8 \mathrm{pW} / \sqrt{ } \mathrm{Hz}$. Channel separation on the detection side is greater than $100 \mathrm{~dB}(20 \log 10)$; however, on the source side due to the construction of the galvo multiplexer, it is only $80 \mathrm{~dB}(20 \log 10)$. Saturation is reached with an input signal of $1.5 \mu \mathrm{W}$, thus combined with the noise equivalent
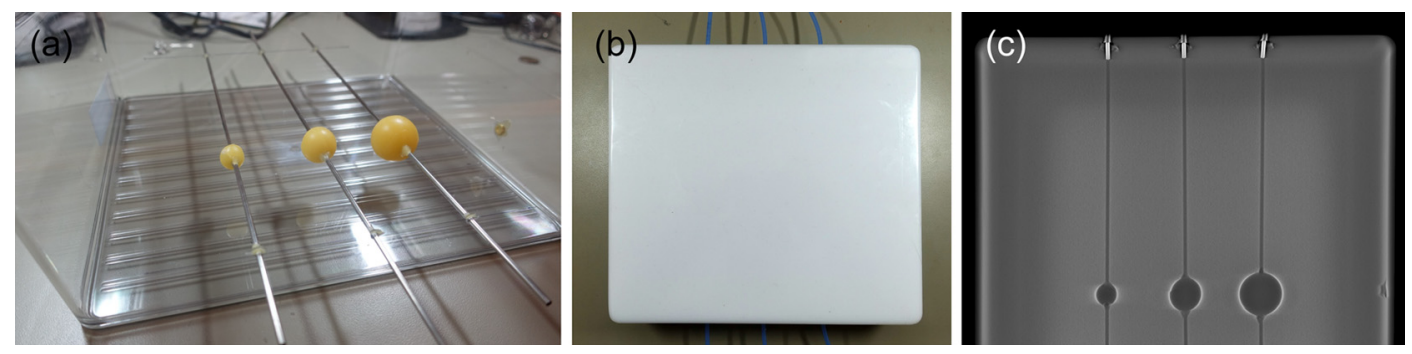

Fig. 3 (a) Mold for the triple-inclusion phantom, with wax balls forming the inclusions mounted on steel tubes. (b) Finished triple-inclusion phantom, with glued-on tubes to fill inclusions with liquid. (c) DBT slice of finished triple-inclusion phantom showing the three spherical cavities with connecting channels. 
power mentioned above, we claim a dynamic range of $115 \mathrm{~dB}$ (20 $\log 10)$. We were not able to observe any interwavelength or amplitude to phase crosstalk. The phase noise of the output signal is smaller than $6 \mathrm{mrad} / \sqrt{ } \mathrm{Hz}$ at 100 -pW input power. Over $10 \mathrm{~h}$, the measured amplitude changes by less than $1.5 \%$, the phase less than $3 \mathrm{mrad}$ at an optical power of $5 \mathrm{nW}$. We also characterized the $\mathrm{CW}$ component (CW6) using the same multiattenuation filter wheel setup as used in Ref. 61 for the RF component. We measured an instantaneous dynamic range of more than $103 \mathrm{~dB}(20 \log 10)$ and a noise floor of $0.06 \mathrm{pW} / \sqrt{ } \mathrm{Hz}$.

To quantify the additional losses incurred due to choosing an x-ray translucent optical probe design, we measured the light transmission of both the source as well as the detector fibers and compared overall throughput versus a simple design where glass fibers directly touch the tissue. On the source side, power is reduced by $3.3 \mathrm{~dB}$, and on the detection side by $6.4 \mathrm{~dB}$ at $830 \mathrm{~nm}$. At $690 \mathrm{~nm}$, the losses are slightly less due to the better transmissivity of the PMMA fibers at this wavelength. The detailed results are shown in Fig. 4. DBT slices of a patient's breast taken with the optical probes in place can be seen in Figs. 5(a)-5(c). In Fig. 5(a), on the upper surface of the breast, the small source fibers are only barely visible, whereas in Fig. 5(b), on the lower surface of the breast, the larger detector fibers and prisms are clearly visible, but their artifacts do not exceed the dynamic range of the x-ray detector. Figure 5(c) shows a center slice, which can be compared to a center slice of the same patient taken without the optical probe attached [Fig. 5(d)]. It is evident that in the center of the breast the DBT reconstruction algorithm already largely removes the artifacts.

\subsection{Static and Dynamic Phantoms}

To test our complete system with all its components, we performed a series of phantom experiments. As a first test, to determine the maximal useable source-detector separation, we measured the single-inclusion phantom described in Sec. 2.5 with the liquid inside the inclusion matched to the bulk optical properties, which in turn are comparable to those of a typical human breast $\left(\mu_{a}=0.075 \mathrm{~cm}^{-1}\right.$ at $690 \mathrm{~nm}$ and $0.052 \mathrm{~cm}^{-1}$ at $830 \mathrm{~nm}, \mu_{s}^{\prime}=8.4 \mathrm{~cm}^{-1}$ at $690 \mathrm{~nm}$ and $7.1 \mathrm{~cm}^{-1}$ at $830 \mathrm{~nm}$ ). The resulting calibrated signal magnitudes versus source-detector separations can be seen in Fig. 6. From this figure, we observe that separations of less than $\sim 9 \mathrm{~cm}$ result in detectable signals above the noise floor, whereas instrument noise dominates in measurements with source-detector separations of more than $9 \mathrm{~cm}$.

To demonstrate the image reconstruction algorithm and evaluate the spatial resolution, we also imaged the triple-inclusion phantom described in Sec. 2.5. All three inclusions were filled with a water, milk, and India ink mixture, with the scattering coefficient matching the bulk of the phantom and the

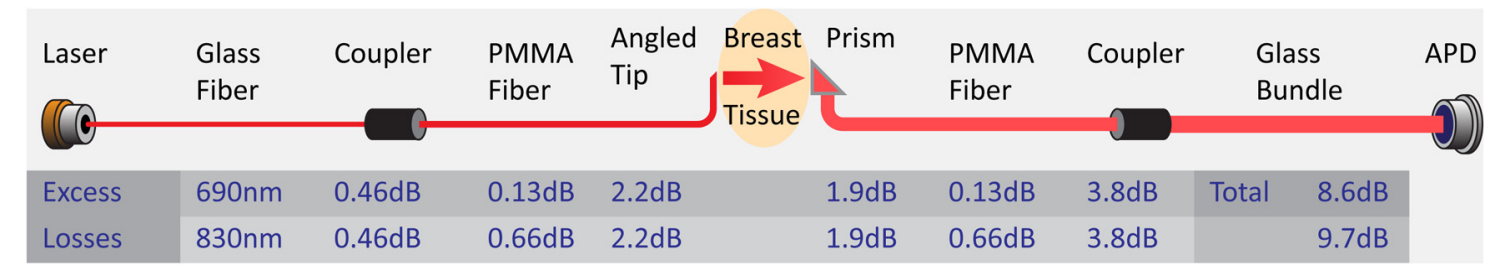

Fig. 4 Schematic showing the additional optical losses incurred due to the hybrid PMMA/glass fiber design versus a minimal fiber-to-tissue approach.
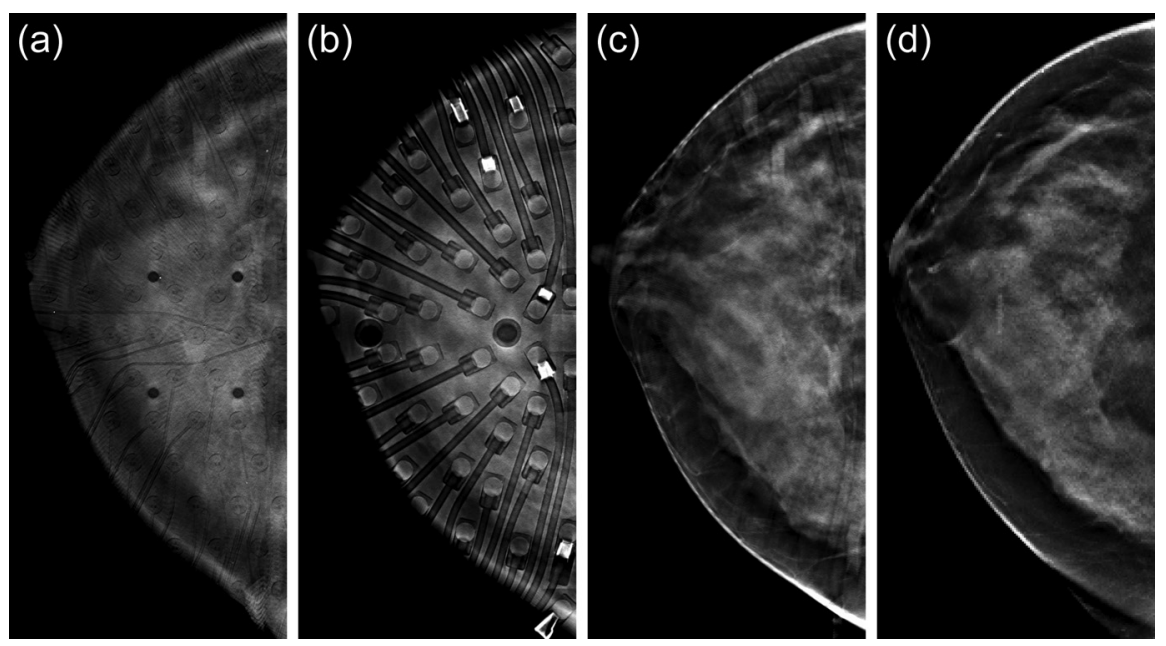

Fig. 5 Impact of optical plates on the DBT images. (a) Top slice of breast DBT volume taken with the optical probe attached. The source fibers and mounting holes are clearly visible. (b) Bottom slice of breast DBT volume taken with the optical probes attached. The detector fibers and prisms are clearly visible. The high absorption patches are the result of small pieces of electrical tape used in the construction of the probe. They were removed subsequently. (c) Middle slice of breast DBT image taken with the optical probe attached. Faint shadows of the detector fibers can be seen. (d) For comparison, middle slice of the separately acquired clinical breast DBT image taken on the same patient (no optical components present). 
absorption coefficient being 1.81 times the value of the bulk at $690 \mathrm{~nm}$. Figure 7(a) shows an absorption image representing the middle slice of the reconstructed 3-D absorption map. The three inclusions can easily be seen, and the centroids are at the expected locations. The reconstructed absorption values at $690 \mathrm{~nm}$ are $0.138,0.142$, and $0.146 \mathrm{~cm}^{-1}$, which represent contrasts of $1.59,1.63$, and 1.68 , respectively, to the reconstructed bulk absorption coefficient of $0.087 \mathrm{~cm}^{-1}$ at $690 \mathrm{~nm}$.

To also test the temporal dynamic performance of the system, the single-inclusion phantom was imaged over $165 \mathrm{~s}$, with one image being reconstructed for every $3 \mathrm{~s}$ of data. During the first third of the measurement period, the water, milk, and India ink mixture in the inclusion was matched to the bulk optical properties both in absorption and scattering. During the middle third of the measurement, the absorption was increased to $1.54 \times$ the baseline value by injecting a different liquid mixture with higher ink concentration into the inclusion. For the last third of the measurement period, the absorption was further increased to $2.82 \times$ of the baseline value. Figure 7 (b) shows an absorption image representing the middle slice of the reconstructed 3-D absorption map during the last third of the measurement. Figure 7(c) shows the time course of the reconstructed absorption values at the center of the inclusion, as well as at the indicated location away from the inclusion for control purposes. The reconstructed absorption value at the control location has an

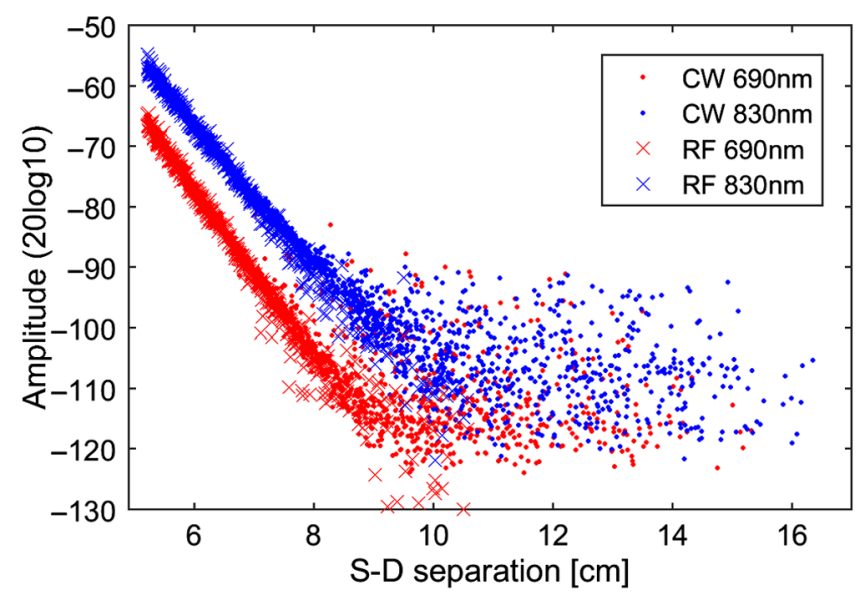

Fig. 6 Plot of the signal amplitude of all possible source-detector combinations versus the corresponding source-detector distances measured in the single inclusion silicone phantom. The optical properties of the inclusion are matched to the bulk, which in turn has properties comparable to a human breast. average of $0.08 \mathrm{~cm}^{-1}$ and stays constant within $\pm 6 \%$. The average values of the reconstructed absorption coefficients at the inclusion centroid during each third of the measurement are $0.072,0.108$, and $0.166 \mathrm{~cm}^{-1}$, respectively. Compared to the targeted $1.54 \times$ and $2.82 \times$ contrast, the reconstructed contrast during the middle and last thirds of the dynamic measurement are $1.5 \times$ and $2.31 \times$, respectively. The two visible spikes are presumably due to an expansion of the cavity due to increased liquid pressure when changing the mixture.

\subsection{Patient Images}

Figures 8(a) and 8(b) show the slice of the total hemoglobin $(\mathrm{HbT})$ concentration map passing through the center of the lesion, overlaid on the corresponding slice of the DBT from the patient scan. Optical images were reconstructed using the adipose and fibro-glandular tissue fractions as priors derived from the x-ray information. ${ }^{63}$ In addition, we used a Gaussian sphere tumor prior with a diameter of $15 \mathrm{~mm}$ at the centroid of the lesion, ${ }^{63}$ as determined by our collaborating radiologist (Saksena). Figure 8(a) shows the absolute HbT concentration and Fig. 8(b) shows the change in HbT due to increasing the compression level from partial to full mammographic force. To facilitate the visibility of hemodynamic changes in the tumor, the color scale in Fig. 8(a) is chosen such that values below $25 \mu \mathrm{M}$, which are representative of normal tissues, are transparent. Similarly, the color scale in Fig. 8(b) is chosen to show positive $\mathrm{HbT}$ changes as transparent. In both images, the tumor area displays localized contrast, increased HbT concentration in the absolute image, and a compression-induced further reduction in $\mathrm{HbT}$ in the relative changes image. Figure 8(c) shows the time course of the mean HbT values in both the tumor region, and in the rest of the breast, respectively. During the first (half-force) compression period, HbT displays a slowly increasing trend in both the tumor and the normal tissues, but the tumor area exhibits what appear to be blood volume oscillations that are not as evident in the normal tissue. The second compression period was rather short, and the distinguishing feature is the substantial decrease in tumor $\mathrm{HbT}$ versus half-compression while only a small further decrease occurs in the normal tissues.

\section{Discussion}

In this paper, we have described and demonstrated our secondgeneration tomographic optical breast imager. One of the main goals of the development of this new instrument was to increase acquisition speed to avoid artifacts due to hemodynamic
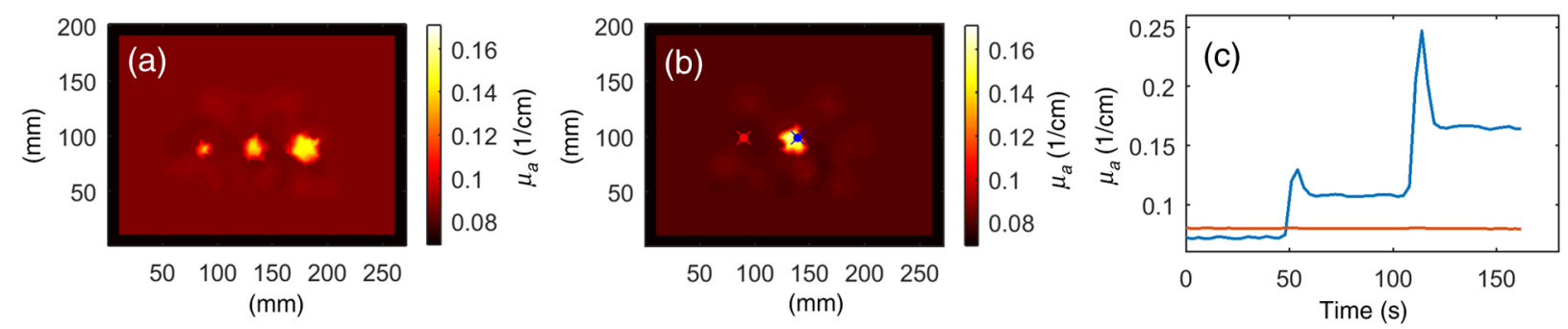

Fig. 7 (a) Middle slice showing the reconstructed absorption map of the triple-inclusion phantom. (b) Middle slice showing the reconstructed absorption map of the single-inclusion phantom during the last third of the measurement. The blue and red markers indicate the location of the corresponding measurements plotted in (c). (c) Time course of the reconstructed absorption coefficients of the singleinclusion phantom at the location of the inclusion (blue), and at a control location (red). 

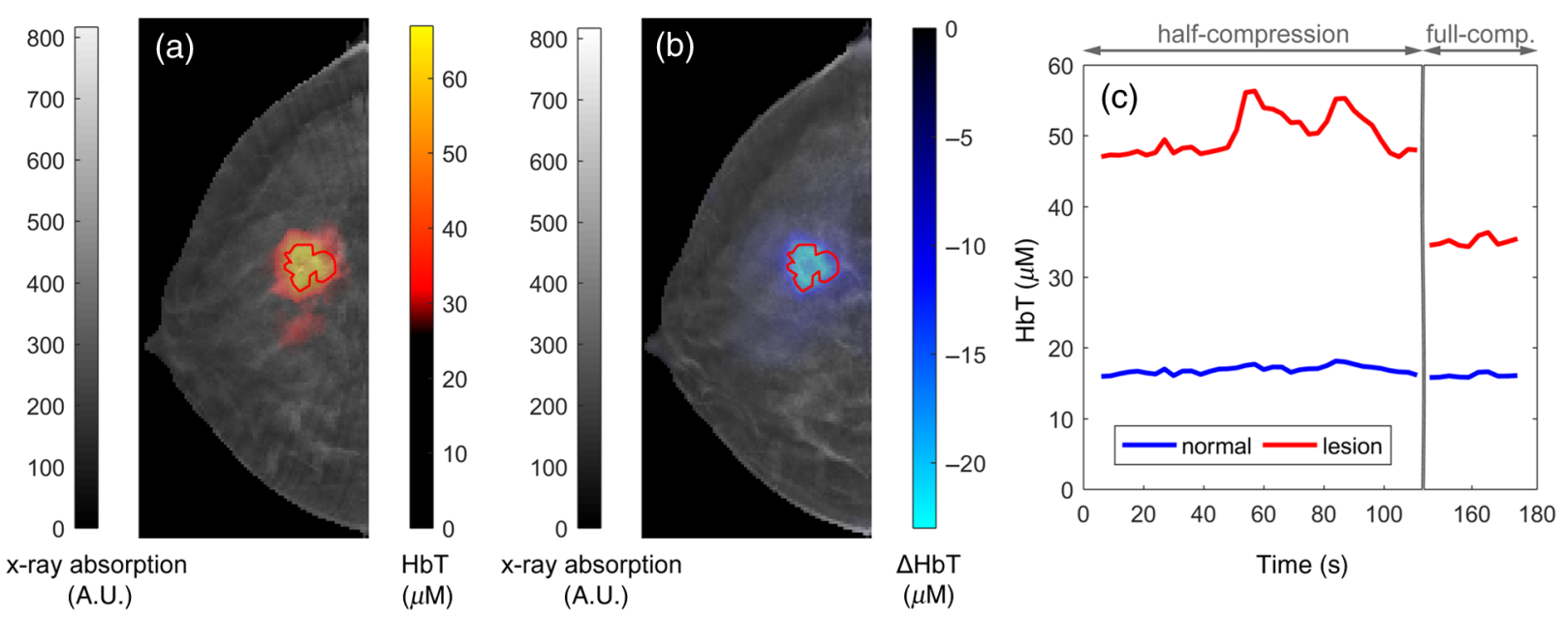

Fig. 8 (a) Absolute HbT concentration map of the slice corresponding to the tumor centroid, overlaid over the corresponding $\mathrm{x}$-ray DBT slice. (b) Change in $\mathrm{HbT}$ concentration as the compression is increased from half to full mammographic force. The red line in (a) and (b) denotes the tumor outline as marked by our collaborating radiologist. (c) The time course of $\mathrm{HbT}$ concentration in the tumor region and normal breast tissue, respectively, during the entire measurement session (the vertical bar and break in the timeline indicate where compression was increased from half to full mammographic compression).

changes during breast compression and in fact capture these dynamics as additional biomarkers. We have achieved this goal by a combination of introducing frequency division multiplexing in the RF component, increasing the switching speed of time division multiplexing for both the CW and the RF component, and using parallel detection channels. Frequency division multiplexing in the RF component was achieved by a direct digital sampling approach previously described by our group in Ref. 61, which allows the instrument to illuminate multiple wavelengths at the same time. Currently, our system is limited to wavelengths of 690 and $830 \mathrm{~nm}$, sufficient to distinguish oxyand deoxy-hemoglobin. Additional wavelengths could be added in the future with minimal supplementary hardware and no decrease in duty cycle. Alternatively, additional lasers at the same wavelengths could also be introduced to further increase parallelism. The switching time between source positions was reduced with a fast galvo-based optical multiplexer. In the $\mathrm{CW}$ component, we have completely eliminated mechanical switching by dedicating a laser to each source fiber and then rotating electronically between laser banks. This approach could also be used in the RF if switching times have to be further reduced to the submillisecond range.

Currently, we have settled on a frame acquisition duration of $3 \mathrm{~s}$, as this seems to represent a good compromise between duty cycle and frame rate. Three seconds per frame is already an order of magnitude faster than our previous system ${ }^{48}$ (and many other breast optical tomography systems). If necessary in the future, we can easily acquire data at faster speeds (e.g., $>1 \mathrm{~Hz}$ ), with a small penalty in signal quality.

The second goal of this new system was to create an x-ray translucent optical probe to enable true simultaneous coregistration of DBT and DOT, which provides improvements in the DOT reconstruction accuracy, DOT and DBT image fusion, as well as reduction in acquisition and breast compression time. To make it possible to leave the optical probe in place during $\mathrm{x}$-ray acquisition, the first priority is to keep its $\mathrm{x}$-ray absorption low and spatially uniform. Added absorption in the x-ray field of view can be compensated up to a certain degree by using a larger dose, but if the probe absorption varies too much, the detector dynamic range can be exceeded, and information will be lost. Glass has a very large x-ray absorption at the energies used for mammography. For example, a single 2.5-mm fiber bundle absorbs approximately as much as the whole breast. For this reason, we decided early on in the design phase to only use plastic fibers. To minimize contrast in both the detection and source side, we embedded the plastic fibers into plastic plates by milling precise channels, thus keeping the $\mathrm{x}$-ray absorption length fairly uniform. Due to the large absorption coefficient of the used PMMA fibers at NIR wavelengths, it was necessary to transition to glass fibers right at the edge of the probe, just outside of the x-ray field of view. As can be seen in Figs. 5(a) and 5(b), artifacts from the probe are visible in the DBT images, but are within the dynamic range of the $\mathrm{x}$ ray detector, so we are confident that they can be removed. In fact, as seen in Fig. 5(c), when compared to Fig. 5(d), the native Hologic DBT image reconstruction algorithm already removes a large fraction of the artifacts. Further improvements could be achieved by performing an image subtraction on raw DBT projection images. The light budget penalty of almost $10 \mathrm{~dB}$ or $90 \%$ might seem excessive, but a quick back of the envelope calculation, using the signal decay profile from Fig. 6, shows that an attenuation by a factor of $10 \times$ only reduces the maximum useful source-detector distance by $\sim 1.3 \mathrm{~cm}$, or $13 \%$. This seems to be an acceptable compromise. The biggest loss results from the transition between detector PMMA fiber to glass fiber bundle, mostly because of the fill factor of the bundle. This could be alleviated in the future by locating the detectors directly at the edge of the probe, making the bundles obsolete. Another option might be the use of perfluorinated fibers, which have a small x-ray and NIR absorption, but currently are very costly and delicate.

We have demonstrated the performance of TOBI2 with raw metrics, phantom tests, as well as initial patient images. The raw performance of both the $\mathrm{CW}$ as well as the RF components improved in every way on our previous system, ${ }^{48}$ and hence exceeded our expectations. The noise floor of both systems 
is close to the photodetector manufacturer specifications, and thus a significant improvement could only be achieved by switching to a different detector like a photo-multiplier tube.

Our phantom results show that we can clearly image inclusions smaller than 13-mm diameter, and dynamic changes are represented correctly. The contrast recovery is up to $\sim 20 \%$ below our expectations, especially for the higher absorption cases. Since this underestimation is also size dependent, with the smaller inclusions experiencing a larger effect, we believe smoothing from the image reconstruction algorithm to be responsible for this, despite our use of a soft prior.

Initial in vivo imaging results are encouraging as well. Values of absolute HbT shown in Fig. 8(a) are consistent with the expected increased HbT contrast of malignant tumors reported by numerous other studies. ${ }^{64,65}$ Dynamic contrast induced by compression changes is also clearly seen in the $\Delta \mathrm{HbT}$ image and time course [Figs. 8(b) and 8(c)], demonstrating the capability of dynamic optical imaging using TOBI2. The tumor dynamic signatures shown here are meant as an example of the data that can be acquired with the TOBI2 system. Group analysis over a larger patient sample is needed to determine whether these signatures are representative. The slow recovery in $\mathrm{HbT}$ as the breast is kept under compression, likely due to the steady decrease in compression force due to tissue relaxation, is consistent with our previous publications. ${ }^{60,66}$ However, due to the complexity of the iterative DBT clinical breast positioning procedure, the optical image acquisition did not begin until $\sim 20$ to $30 \mathrm{~s}$ after the breast tissue initially experienced compression. As a result, the early response is not captured in the presented data. Previously, using a standalone dynamic optical imaging system with a computer controlled compression mechanism, we have seen an early decrease in tumor $\mathrm{HbT}$ during compression. ${ }^{60,66}$ However, in the example TOBI2 scan, this early decrease has likely occurred before the measurement was initiated (while the radiology technician was positioning the breast under compression to match clinical standards). The significantly larger decrease in tumor HbT observed after the transition to full compression versus the surrounding normal tissue (likely related to the higher stiffness of tumor tissue) may be a useful tumor marker and will be further characterized in future work.

These encouraging results give us confidence that combining dynamic optical imaging with $\mathrm{x}$-ray DBT in true simultaneous coregistration can provide a reliable platform for both breast cancer detection and chemotherapy guidance. Additional possible improvements are the addition of extra wavelengths to the RF component, the optimization of the acquisition software, automation of x-ray artifact removal, and improving the DOT reconstruction algorithm by incorporating temporal regularization. Also, the long-term durability of the plastic optical fiber probe remains to be explored.

\section{Conclusion}

In this work, we have presented a fast, hybrid tomographic optical breast imaging system featuring $3072 \mathrm{CW}$ channels (96 CW sources $\times 32 \mathrm{CW}$ detectors), $480 \mathrm{RF}$ channels (24 RF sources $\times$ $20 \mathrm{RF}$ detectors), up to $1 \mathrm{~Hz}$ acquisition rate, and an X-ray translucent probe. Tests show that our second-generation system exceeds the basic performance metrics of our previous optical-DBT system and meets our expectations. Using phantoms with glass-free inclusions, we demonstrate that we can image features of less than 13-mm diameter with good quantitative accuracy by employing soft-prior constrained reconstruction and capture their dynamics. DBT images taken with the optical probe attached show that the resulting artifacts are small enough for us to be confident that it will be possible to remove them automatically in the future. First patient images testify to the usefulness of these new features. These results pave the way for future clinical studies, in conjunction with improvements in our data acquisition interface and image reconstruction pipeline.

\section{Disclosures}

No conflicts of interest, financial or otherwise, are declared by the authors.

\section{Acknowledgments}

This work was funded by the National Institutes of Health (Grant Nos. R01-CA187595, R01-CA142575, R01CA097305, and R00-EB011889). The authors would like to thank Joe Stadtmiller, Robert Dewsnap, Ron Jose, Ron Altman, William Johnson, and Arthur DiMartino at TechEn Inc. for collaborative efforts in developing the instrumentation.

\section{References}

1. R. L. Siegel, K. D. Miller, and A. Jemal, "Cancer statistics, 2016," CA Cancer J. Clin. 66(1), 7-30 (2016).

2. H. D. Nelson et al., "Screening for breast cancer: an update for the US preventive services task force," Ann. Intern. Med. 151(10), 727-737, W237-W242 (2009).

3. K. Kerlikowske et al., "Effect of age, breast density, and family history on the sensitivity of first screening mammography," J. Am. Med. Assoc. 276(1), 33-38 (1996).

4. R. D. Rosenberg et al., "Effects of age, breast density, ethnicity, and estrogen replacement therapy on screening mammographic sensitivity and cancer stage at diagnosis: review of 183, 134 screening mammograms in Albuquerque, New Mexico," Radiology 209(2), 511-518 (1998).

5. E. A. Rafferty et al., "Assessing radiologist performance using combined digital mammography and breast tomosynthesis compared with digital mammography alone: results of a multicenter, multireader trial," Radiology 266(1), 104-113 (2013).

6. E. D. Pisano et al., "Diagnostic performance of digital versus film mammography for breast-cancer screening," N. Engl. J. Med. 353(17), 17731783 (2005).

7. D. B. Kopans, "The positive predictive value of mammography," Am. J. Roentgenol. 158(3), 521-526 (1992).

8. P. Skaane et al., "Comparison of digital mammography alone and digital mammography plus tomosynthesis in a population-based screening program," Radiology 267(1), 47-56 (2013).

9. M. L. Zuley et al., "Digital breast tomosynthesis versus supplemental diagnostic mammographic views for evaluation of noncalcified breast lesions," Radiology 266(1), 89-95 (2013).

10. S. M. Friedewald et al., "Breast cancer screening using tomosynthesis in combination with digital mammography," J. Am. Med. Assoc. 311(24), 2499-2507 (2014).

11. L. T. Niklason et al., "Digital tomosynthesis in breast imaging," Radiology 205(2), 399-406 (1997).

12. H.-P. Chan et al., "Computer-aided detection system for breast masses on digital tomosynthesis mammograms: preliminary experience," Radiology 237(3), 1075-1080 (2005).

13. D. B. Kopans, "Digital breast tomosynthesis from concept to clinical care," Am. J. Roentgenol. 202(2), 299-308 (2014).

14. H. R. Peppard et al., "Digital breast tomosynthesis in the diagnostic setting: indications and clinical applications," Radiographics 35(4), 975-990 (2015).

15. S. Vedantham et al., "Digital breast tomosynthesis: state of the art," Radiology 277(3), 663-684 (2015). 
16. M. Kriege et al., "Efficacy of MRI and mammography for breast-cancer screening in women with a familial or genetic predisposition," N. Engl. J. Med. 351(5), 427-437 (2004).

17. E. Warner et al., "Surveillance of BRCA1 and BRCA2 mutation carriers with magnetic resonance imaging, ultrasound, mammography, and clinical breast examination," J. Am. Med. Assoc. 292(11), 1317-1325 (2004).

18. M. O. Leach et al., "Screening with magnetic resonance imaging and mammography of a UK population at high familial risk of breast cancer: a prospective multicentre cohort study (MARIBS)," Lancet Lond. Engl. 365(9473), 1769-1778 (2005).

19. C. D. Lehman et al., "Screening women at high risk for breast cancer with mammography and magnetic resonance imaging," Cancer 103(9), 1898-1905 (2005).

20. A. K. Narayan, K. Visvanathan, and S. C. Harvey, "Comparative effectiveness of breast MRI and mammography in screening young women with elevated risk of developing breast cancer: a retrospective cohort study," Breast Cancer Res. Treat. 158(3), 583-589 (2016).

21. N. Y. Tse et al., "The application of positron emission tomographic imaging with fluorodeoxyglucose to the evaluation of breast disease," Ann. Surg. 216(1), 27-34 (1992).

22. L. P. Adler et al., "Evaluation of breast masses and axillary lymph nodes with [F-18] 2-deoxy-2-fluoro-D-glucose PET," Radiology 187(3), 743750 (1993).

23. G. W. Görres, H. C. Steinert, and G. K. von Schulthess, "PET and functional anatomic fusion imaging in lung and breast cancers," Cancer J. 10(4), 251-261 (2004).

24. K. Murthy et al., "Results of preliminary clinical trials of the positron emission mammography system PEM-I: a dedicated breast imaging system producing glucose metabolic images using FDG," J. Nucl. Med. 41(11), 1851-1858 (2000).

25. E. A. Levine et al., "Positron emission mammography: initial clinical results," Ann. Surg. Oncol. 10(1), 86-91 (2003).

26. E. L. Rosen et al., "Detection of primary breast carcinoma with a dedicated, large-field-of-view FDG PET mammography device: initial experience," Radiology 234(2), 527-534 (2005).

27. V. Kalles et al., "The current status of positron emission mammography in breast cancer diagnosis," Breast Cancer 20(2), 123-130 (2013).

28. C. Caldarella, G. Treglia, and A. Giordano, "Diagnostic performance of dedicated positron emission mammography using fluorine-18fluorodeoxyglucose in women with suspicious breast lesions: a meta-analysis," Clin. Breast Cancer 14(4), 241-248 (2014).

29. R. E. Hendrick, "Radiation doses and cancer risks from breast imaging studies," Radiology 257(1), 246-253 (2010).

30. T. Durduran et al., "Diffuse optics for tissue monitoring and tomography," Rep. Prog. Phys. 73(7), 076701 (2010).

31. B. W. Pogue et al., "Quantitative hemoglobin tomography with diffuse near-infrared spectroscopy: pilot results in the breast," Radiology 218(1), 261-266 (2001).

32. N. Iftimia et al., "A compact, parallel-detection diffuse optical mammography system," Rev. Sci. Instrum. 74(5), 2836-2842 (2003).

33. Y. Zhao et al., "Portable, parallel 9-wavelength near-infrared spectral tomography (NIRST) system for efficient characterization of breast cancer within the clinical oncology infusion suite," Biomed. Opt. Express 7(6), 2186-2201 (2016).

34. C. H. Schmitz et al., "Design and implementation of dynamic nearinfrared optical tomographic imaging instrumentation for simultaneous dual-breast measurements," Appl. Opt. 44(11), 2140-2153 (2005).

35. J. P. Culver et al., "Three-dimensional diffuse optical tomography in the parallel plane transmission geometry: evaluation of a hybrid frequency domain/continuous wave clinical system for breast imaging," Med. Phys. 30(2), 235-247 (2003).

36. R. Choe et al., "Differentiation of benign and malignant breast tumors by in-vivo three-dimensional parallel-plate diffuse optical tomography," J. Biomed. Opt. 14(2), 024020 (2009).

37. H. Y. Ban et al., "Heterodyne frequency-domain multispectral diffuse optical tomography of breast cancer in the parallel-plane transmission geometry," Med. Phys. 43(7), 4383-4395 (2016).

38. A. Li et al., "Tomographic optical breast imaging guided by threedimensional mammography," Appl. Opt. 42(25), 5181-5190 (2003).

39. B. Brooksby et al., "Combining near-infrared tomography and magnetic resonance imaging to study in vivo breast tissue: implementation of a
Laplacian-type regularization to incorporate magnetic resonance structure," J. Biomed. Opt. 10(5), 051504 (2005).

40. L. K. Dunnwald et al., "Tumor metabolism and blood flow changes by positron emission tomography: relation to survival in patients treated with neoadjuvant chemotherapy for locally advanced breast cancer," J. Clin. Oncol. 26(27), 4449-4457 (2008).

41. R. M. L. Warren et al., "Can breast MRI help in the management of women with breast cancer treated by neoadjuvant chemotherapy?" Br. J. Cancer 90(7), 1349-1360 (2004).

42. Q. Zhu et al., "Early-stage invasive breast cancers: potential role of optical tomography with US localization in assisting diagnosis," Radiology 256(2), 367-378 (2010).

43. F. El-Ghussein et al., "Hybrid photomultiplier tube and photodiode parallel detection array for wideband optical spectroscopy of the breast guided by magnetic resonance imaging," J. Biomed. Opt. 19(1), 011010 (2014).

44. V. Krishnaswamy et al., "A digital x-ray tomosynthesis coupled near infrared spectral tomography system for dual-modality breast imaging," Opt. Express 20(17), 19125 (2012).

45. S. Vedantham et al., "Digital breast tomosynthesis guided near infrared spectroscopy: volumetric estimates of fibroglandular fraction and breast density from tomosynthesis reconstructions," Biomed. Phys. Eng. Express 1(4), 045202 (2015).

46. K. E. Michaelsen et al., "Calibration and optimization of 3D digital breast tomosynthesis guided near infrared spectral tomography," Biomed. Opt. Express 6(12), 4981-4991 (2015).

47. Q. Zhang et al., "Coregistered tomographic $\mathrm{x}$-ray and optical breast imaging: initial results," J. Biomed. Opt. 10(2), 024033 (2005).

48. Q. Fang et al., "Combined optical imaging and mammography of the healthy breast: optical contrast derived from breast structure and compression," IEEE Trans. Med. Imaging 28(1), 30-42 (2009).

49. Q. Fang et al., "Combined optical and x-ray tomosynthesis breast imaging," Radiology 258(1), 89-97 (2011).

50. M. L. Flexman et al., "Digital optical tomography system for dynamic breast imaging," J. Biomed. Opt. 16(7), 076014 (2011).

51. M. L. Flexman et al., "Optical biomarkers for breast cancer derived from dynamic diffuse optical tomography," J. Biomed. Opt. 18(9), 096012 (2013).

52. C. M. Carpenter et al., "Inspired gas-induced vascular change in tumors with magnetic-resonance-guided near-infrared imaging: human breast pilot study," J. Biomed. Opt. 15(3), 036026 (2010).

53. R. X. Xu et al., "A prospective pilot clinical trial evaluating the utility of a dynamic near-infrared imaging device for characterizing suspicious breast lesions," Breast Cancer Res. 9(6), R88 (2007).

54. B. Wang et al., "Dynamic schema for near infrared detection of pressure-induced changes in solid tumors," Appl. Opt. 47(16), 3053-3063 (2008).

55. L. S. Fournier et al., "Dynamic optical breast imaging: a novel technique to detect and characterize tumor vessels," Eur. J. Radiol. 69(1), 43-49 (2009).

56. R. Al abdi et al., "Optomechanical imaging system for breast cancer detection," J. Opt. Soc. Am. A 28(12), 2473-2493 (2011).

57. H. L. Graber et al., "Enhanced resting-state dynamics of the hemoglobin signal as a novel biomarker for detection of breast cancer," Med. Phys. 42(11), 6406-6424 (2015).

58. S. A. Carp et al., "Compression-induced changes in the physiological state of the breast as observed through frequency domain photon migration measurements," J. Biomed. Opt. 11(6), 064016 (2006).

59. S. A. Carp et al., "Dynamic functional and mechanical response of breast tissue to compression," Opt. Express 16(20), 16064 (2008).

60. S. A. Carp et al., "Hemodynamic signature of breast cancer under fractional mammographic compression using a dynamic diffuse optical tomography system," Biomed. Opt. Express 4(12), 2911-2924 (2013).

61. B. B. Zimmermann et al., "Frequency domain near-infrared multiwavelength imager design using high-speed, direct analog-to-digital conversion," J. Biomed. Opt. 21(1), 016010 (2016).

62. Q. Fang, "iso2mesh: a 3D surface and volumetric mesh generator for MATLAB/Octave," http://iso2mesh.sourceforge.net/cgi-bin/index.cgi (17 October 2016).

63. B. Deng et al., "Characterization of structural-prior guided optical tomography using realistic breast models derived from dual-energy x-ray mammography," Biomed. Opt. Express 6(7), 2366-2379 (2015). 
64. Q. Zhu et al., "Assessment of functional differences in malignant and benign breast lesions and improvement of diagnostic accuracy by using US-guided diffuse optical tomography in conjunction with conventional US," Radiology 280(2), 387-397 (2016).

65. B. J. Tromberg et al., "Predicting responses to neoadjuvant chemotherapy in breast cancer: ACRIN 6691 trial of diffuse optical spectroscopic imaging," Cancer Res. 76(20), 5933-5944 (2016).
66. A. Y. Sajjadi et al., "Normalization of compression-induced hemodynamics in patients responding to neoadjuvant chemotherapy monitored by dynamic tomographic optical breast imaging (DTOBI)," Biomed. Opt. Express 8(2), 555-569 (2017).

Biographies for the authors are not available. 